\title{
Evaluation of spin labels for in-cell EPR by analysis of nitroxide reduction in cell extract of Xenopus laevis oocytes
}

\author{
Mykhailo Azarkh ${ }^{*, 1}$, Oliver Okle ${ }^{1}$, Philipp Eyring, Daniel R. Dietrich, Malte Drescher \\ University of Konstanz, Departments of Chemistry and Biology, Konstanz Research School Chemical Biology (KoRS-CB), Zukunftskolleg, 78457 Konstanz, Germany
}

\begin{abstract}
A B S T R A C T
Spin-label electron paramagnetic resonance (SL-EPR) spectroscopy has become a powerful and useful tool for studying structure and dynamics of biomacromolecules. However, utilizing these methods at physiological temperatures for in-cell studies is hampered by reduction of the nitroxide spin labels and thus short half-lives in the cellular environment. Consequently, reduction kinetics of two structurally different nitroxides was investigated in cell extracts of Xenopus laevis oocytes using rapid-scan cw-experiments at X-band. The five member heterocyclic ring nitroxide PCA (3-carboxy-2,2,5,5-tetramethylpyrrolidinyl-1-oxy) under investigation features much higher stability against intracellular reduction than the six member ring analog TOAC (2,2,6,6-tetramethylpiperidine-N-oxyl-4-amino-4-carboxilic acid) and is therefore a suitable spin label type for in-cell EPR. The kinetic data can be described according to the Michaelis-Menten model and thus suggest an enzymatic or enzyme-mediated reduction process.
\end{abstract}

\section{Introduction}

EPR, first introduced by Zavoisky [1] for transition metal complex, is based on the intrinsic magnetic moment of unpaired electrons and thus is suitable for all systems where species carrying unpaired electron(s) are present, can be produced, or introduced externally. Since the 1950 s biological problems were addressed with EPR $[2,3]$. Nowadays this method allows, especially in conjunction with spin labeling strategies, the characterization of the environment in terms of viscosity [5], partial pressure of oxygen [4], polarity [6] and $\mathrm{pH}[7]$, study of dynamics [8] and redox kinetics [9], as well as determination of structure or structural changes of nucleic acids [10], peptides [11], proteins [12] and viruses [13] in solution.

The non-invasive nature of EPR spectroscopy strongly supports its application for investigation of living systems and living organisms. While in vivo EPR is restricted to low frequencies (300-1200 MHz) and only small animals (mice) [14,15], emerging in-cell EPR [16-18] has no such limitations and can be performed on various types of cells in a standard EPR tube at X-band. Compared to in-cell NMR $[19,20]$, in-cell EPR has two main advantages: Lower concentrations of spin labels can be used because EPR is much more sensitive per spin than NMR, and, since EPR only detects unpaired electron spins, no background from diamagnetic molecules is observed. The latter is of particular importance since, in contrast to structural studies of isolated macromolecules, in-cell

\footnotetext{
* Corresponding author. Fax: +49 7531883139.

E-mail address: mykhailo.azarkh@uni-konstanz.de (M. Azarkh)

${ }^{1}$ Authors contributed equally to this work.
}

experiments are often hampered by many different cellular components thereby providing for high background signals. On the other hand, in-cell studies are of greater significance as, in contrast to pure solution experiments, a natural environment is provided which may be crucial for observing the biologically relevant conformation of biomacromolecules $[21,22]$.

Oocytes from African frog Xenopus laevis (X. laevis) are widely used model systems in cell- and development-biology. Indeed, there are numerous studies on the regulation of $\mathrm{mRNA}$ and the heterologous expression of proteins e.g. ion channels, transporters and receptors [23-26]. Consequently, there are several published techniques that allow the analysis of macromolecular complex formation and metabolism up to an exposure period of 6 days $[27,28]$. Nitroxide labeled proteins or nucleic acids can be delivered into these cells via microinjection [29].

In fact, a large body of results on nitroxides - which are suitable spin probes and widely used spin labels due to their small size and high stability in aqueous solution - in different cells is reported and are reviewed in [30]. These reports cover oximetry in intraand extracellular environments, problems of penetration of nitroxides through cell membranes, pharmacokinetic studies of spin labeled drugs, and stability of nitroxides inside isolated cells. The reduction of nitroxides and thus short half-lives in the cellular environment however limit their potential application for in-cell EPR. Shock-freezing of the sample, as routinely used for distance measurements, can prevent spin label reduction but in turn precludes in-cell EPR application. Indeed, in-cell EPR applications demand longer incubation time at physiological temperatures, especially where slow folding kinetics are involved. 
Two recent publications on subject of in-cell distance measurements on nucleic acids utilizing pulsed EPR demonstrated general possibility to use spin labels of both types: including five or six member ring $[17,18]$. The stability of five member ring nitroxides in the reductive environment of oocytes is higher in comparison to six member ring one and this is in agreement with observation in other cell types [30]. Although the six member ring spin labels can be used for in-cell application, these types of experiments are restricted to very short incubation times. And respective stability studies of nitroxides in oocytes were limited to establishing halflives only $[16-18]$.

The present manuscript deals with a deeper characterization of kinetic aspects of the stability of two structurally different nitroxides - representing two commonly used types of spin labels - in the cell extracts from $X$. laevis oocytes using rapid-scan $c W$-EPR experiment at X-band. The quantitative analysis of the reduction kinetics suggests an enzymatic origin of the spin label reduction.

\section{Results}

Cell extract from $X$. laevis oocytes was used for assessing intracellular nitroxide spin label reduction. PCA and TOAC nitroxides (Fig. 1) differing in size of the heterocyclic ring were chosen for the comparative reduction studies. PCA and TOAC nitroxide are comparable to other widely used five or six membered ring nitroxide spin labels e.g. MTSSL [11] and TEMPA [31], respectively. For solubility reasons, PCA and TOAC were used as their $\mathrm{K}^{+}$salts. Using $X$. laevis oocyte cell free cytoplasm extract allowed for better manipulation and real-time observation of macromolecules involved in the cell cycle and signaling pathways under nearly "in-cell" conditions [32-34].

The cw-EPR spectra of both nitroxides in the oocyte extract displayed a triplet due to hyperfine coupling with the nitrogen atom (Fig. S1). Rotational correlation times calculated for these spectra are slightly longer than for spectra recorded in aqueous solution, thereby reflecting the higher viscosity of the cell extract.

To monitor the reduction kinetics of the nitroxides, spectra for each individual sample were acquired time-dependently. The amount of non-reduced PCA was determined by the amplitude of the low-field peak and plotted versus time (Fig. 2a). For determination of non-reduced TOAC species double integration of the whole spectrum was performed. The latter provides reliable estimation of amounts of EPR active species for samples with broadened lines, as was the case for high TOAC concentrations where the peak amplitude is no longer a correct criterion for active species quantification.

Initial rates for the reduction reaction $V$ were determined as slope in the linearly approximated region of decay curves (Fig. 2), where the decay does not exceed $20 \%$, and were plotted versus initial concentration of the nitroxides (Fig. 3). Full saturation for PCA was observed in the range of $2.5-4 \mathrm{mM}$. In contrast, full

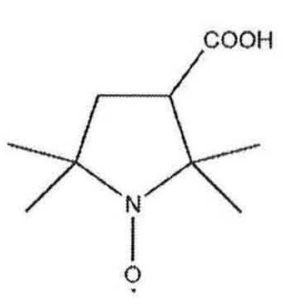

PCA

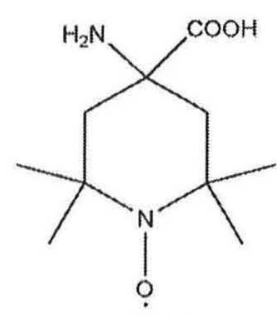

TOAC
Fig. 1. Chemical schemes of nitroxides used for study in cell extract from $X$. laevis oocytes. saturation was not achieved for TOAC, albeit the curve shown in Fig. 3 b does suggest approximation to saturation at a concentration $\geqslant 20 \mathrm{mM}$. Unfortunately, no reasonable experimental characterization appeared possible at concentrations $>20 \mathrm{mM}$, as use of highly concentrated TOAC stock solutions gave rise to extremely broadened EPR lines. The latter was due to interactions between nitroxides and improper mixing of the solutions within the short experimental times required.

Kinetic data were fitted according to the Michaelis-Menten model for enzymatic processes [35] (Eq. (2), see below). Respective constants of $K_{M}$ and $V_{\max }$ were extracted from the fits (Table 1) and are discussed below.

Incubation of the $X$. laevis oocyte extracts at room temperature for $4 \mathrm{~h}$ before mixing it with a nitroxide avoided reduction of the nitroxide spin labels. Highly concentrated (several molar) alkali solutions inhibited nitroxide reduction as well.

\section{Discussion}

As proposed by Swartz et al. [36] reduction of nitroxides inside cells most likely has an enzymatic origin, while reduction in an extracellular environment is due to the amount of ascorbate present. The purpose of our work was a systematic study of the reduction of nitroxides in the cellular environment of $X$. laevis oocytes, which is of particular interest for spin labeling strategies in in-cell EPR. The slower decay of the EPR signal for PCA (Fig. 2a) allowed decay monitoring with conventional $\mathrm{cW}$-EPR experiments and thus provided for very small SEM. (Fig. 3a). In contrast, the faster decay of the TOAC signal allowed monitoring with a rapid scan technique only. Thus with initial reduction rates $V$ of TOAC less data points per decay were available, thereby resulting in larger SEM. for the initial rates (compare $V$ at low and high TOAC concentrations in Fig. 3b).

A tendency toward saturation of the reaction rates depicted for plots of PCA and TOAC (Fig. 3) indicates that the reaction order changes from one to zero, which is characteristic for enzymatic kinetics [37]. Assuming enzymatic origin of the reduction of nitroxides in the cell extract of $X$. laevis oocytes, the obtained kinetic data were analyzed using the Michaelis-Menten model for enzymatic processes. This model considers reversible formation of a transitional enzyme-substrate complex with the subsequent conversion of the substrate to a product:

$E+S \underset{k_{-1}}{\stackrel{k_{1}}{\rightleftharpoons}} E S \stackrel{k_{2}}{\rightarrow} E+P$

where $E$ denotes enzyme, $S$ - substrate, $P$ - product, and $k_{i}$ are the corresponding rate constants. In our case the nitroxide molecule corresponds to the substrate $S$ and its reduced form to product $P$.

The overall reaction rate is given by:

$V=\frac{V_{\max }[S]}{[S]+K_{M}}$

where the maximal reaction rate $V_{\max }$ and the Michaelis constant $K_{M}$ are defined as follows:

$V_{\max }=k_{2}[E]_{0}$

$K_{M}=\frac{k_{-1}+k_{2}}{k_{1}}$

The $V_{\max }$ values for PCA and TOAC reduction in the cell extracts were compared with each other (Table 1 ). The maximal reduction rate for TOAC appeared to be more than 650 times higher than for PCA. According to Eq. (3), the maximal reduction rate is proportional to the rate constant $k_{2}$ and initial enzyme concentration. Under assumption that the same enzymes take part in the reduction 

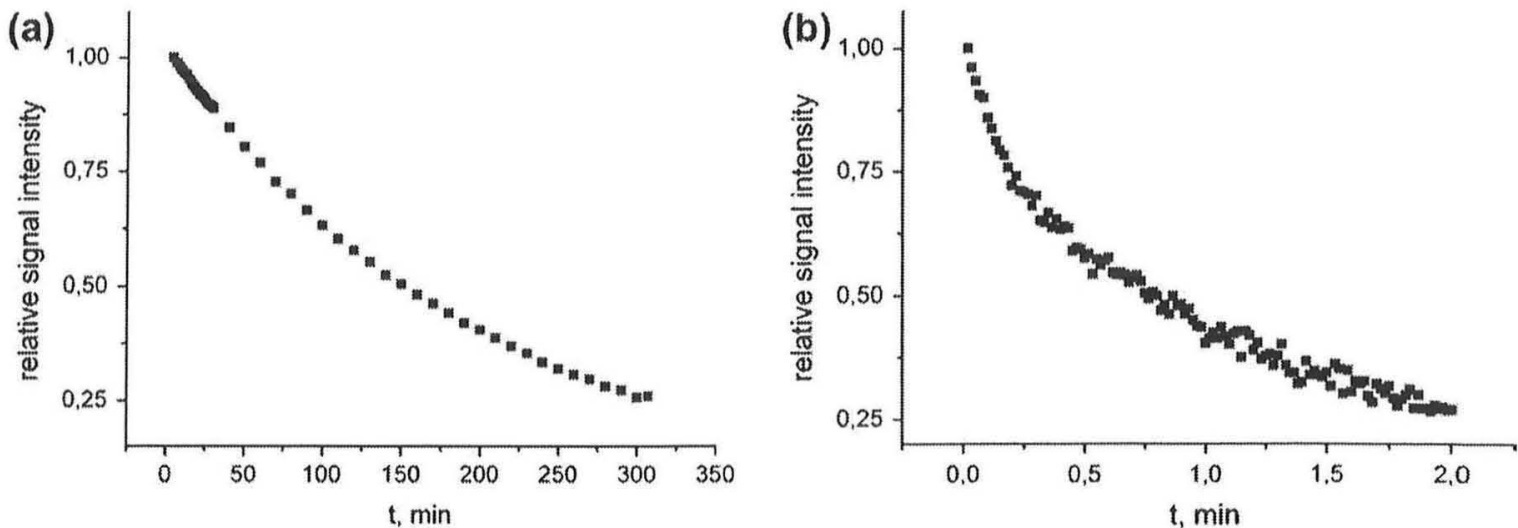

Fig. 2. Example decay curves of the nitroxide signal in the cell extracts at $18^{\circ} \mathrm{C}$ : (a) $2 \mathrm{mM}$ solution of PCA, (b) 2 mM solution of TOAC.
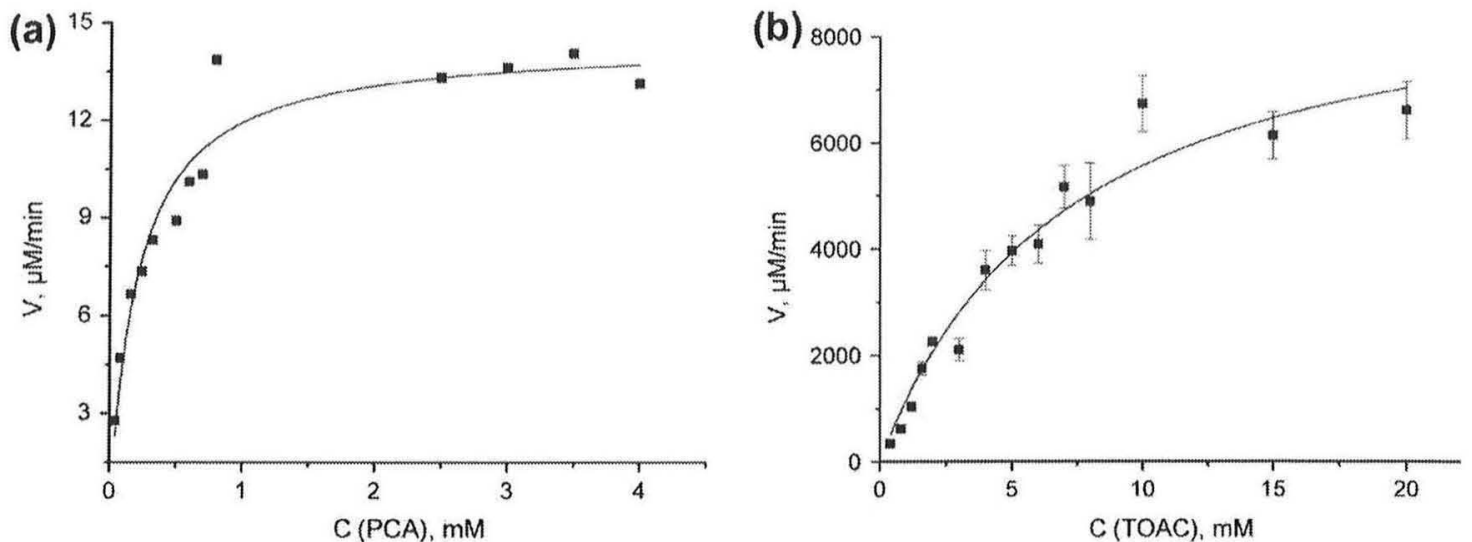

Fig. 3. Michaelis-Menten plots for the reduction kinetics of PCA (a) and TOAC (b) in oocyte extracts at $18^{\circ} \mathrm{C}$. Each data point represents the mean value of three parallel measurements ( \pm standard error of means (SEM)). Note: SEM in the PCA plot (a) are so small they do not exceed the corresponding data symbols in the plot.

Table 1

$V_{\text {max }}$ and $K_{M}$ values for reduction kinetics of PCA and TOAC in X. laevis oocyte extracts at $T=18^{\circ} \mathrm{C}$.

\begin{tabular}{lcl}
\hline Nitroxide & $V_{\max } \pm \operatorname{SEM}(\mu \mathrm{M} / \mathrm{min})$ & $K_{M} \pm \operatorname{SEM}(\mathrm{mM})$ \\
\hline PCA & $14.4 \pm 0.1$ & $0.21 \pm 0.03$ \\
TOAC & $9600 \pm 960$ & $7.21 \pm 1.50$ \\
\hline
\end{tabular}

of PCA and TOAC, the enzyme concentration can be assumed to be equal for the PCA and TOAC experiments as the same extract was used for all measurements. Thus, the ratio between $V_{\max }$ for the two nitroxides corresponds to the ratio between their rate constants, that is: $k_{2}(\mathrm{PCA}) / k_{2}$ (TOAC) $=1 / 667$.

The higher $k_{2}$ value for TOAC is also reflected in the Michaelis constant which is determined by the enzyme-substrate affinity and the enzyme mediated substrate specific reduction rate $\left(k_{2}\right)$. Hence, although no conclusions can be drawn concerning the affinity between the enzyme and the different nitroxides, a dominant role of $k_{2}$ in the overall reduction rate of nitroxides could be established.

The Michaelis-Menten process is not applicable without restrictions, that is for many enzyme-mediated reactions which consist of several intermediate steps. Indeed, determining the maximal reaction rate $V_{\max }$ and the Michaelis constant $K_{M}$ does not allow for identifying such a multistep enzyme-mediated reaction [38]. Thus, based on our data a multistep enzyme-mediated nitroxides reduction process in cellulo cannot be excluded.
Consequently, the term "enzyme-mediated" represents all enzyme mediated reductions whereby we do not discriminate between direct reduction by enzymes and enzymatic production of a reducing intermediate which diffuses to the nitroxide [9].

To prolong half-lives of nitroxides in a cellular environment, selective and non-selective inactivation of enzymes mediating the reduction process can be used. However, selective inhibition of the enzyme or regulation of its affinity to the nitroxide requires some knowledge about the particular enzyme. Simple approaches for inactivation are incubation of cells at room temperature before microinjection or using high concentrated alkali buffers deactivating reduction of nitroxides, but they can in turn influence other processes in cellulo. Further study and understanding of nitroxides reduction kinetics in the cellular environment could help to achieve significant increase in half-lives of nitroxide spin labels and to enable in-cell EPR distance measurements where longer equilibration of spin labeled samples inside cells is of ultimate importance.

\section{Conclusions}

Nitroxide spin label stability primarily depends on the size of the heterocyclic ring. For the first time comparative kinetic analysis of nitroxides' reduction in cell extracts of $X$. laevis oocytes was performed. PCA and TOAC nitroxides are different in their resistance towards reduction in cellulo, and a reduction process is shown to be an enzyme-mediated one. Consequently, the kinetic 
data can be described using the Michaelis-Menten model and allow determination of $V_{\max }$ and $K_{M}$ constants. Simple approaches for enzyme inactivation and prolongation of half-lives of nitroxides were suggested. Upon proper choice of the nitroxide spin label, incell EPR distance measurements which need long incubation times after microinjection, e.g. to reach an equilibrium state, will become possible. Thus using in-cell EPR and the appropriate nitroxide spin labels opens new avenues to study the structure of biomacromolecules under native conditions.

\section{Experimental}

\subsection{Chemicals and cell extract}

Nitroxides 3-carboxy-2,2,5,5-tetramethylpyrrolidinyl-1-oxy (PC A) and 2,2,6,6-tetramethylpiperidine-N-oxyl-4-amino-4-carboxilic acid (TOAC), purchased from Toronto Research Chemicals Inc (Canada), were dissolved in Milli-Q water with equivalent amount of $\mathrm{KOH}$ (Sigma-Aldrich). Cell extract from X. laevis oocytes was prepared as stated in SI, frozen and stored at $-20^{\circ} \mathrm{C}$ until further use (it was taken from the freezer and thawed immediately prior sample preparation).

\subsection{Reduction kinetics of PCA}

Slow reduction kinetics of PCA was followed on the Miniscope MS200 spectrometer operating at X-band $(9.4 \mathrm{GHz})$ equipped with the TC-H02 temperature controller (Magnettech). Each individual sample was prepared by mixing $8 \mu \mathrm{L}$ of cell extract with $2 \mu \mathrm{L}$ of PCA and measured in capillaries (Magnettech) varying the nitroxide concentration in the range from 0.04 to $4 \mathrm{mM}$. For one kinetic curve the sample was monitored over $15 \mathrm{~min}$ ( 1 spectrum $/ \mathrm{min}$ ). Time-dependent automatic acquisition was performed using Autolt $v 3$ Software. The amount of nitroxide species in the probe was determined exploiting the height of the low-field peak.

\subsection{Reduction kinetics of TOAC}

Fast reduction kinetics of TOAC was followed on the ELEXSYS E580 spectrometer (Bruker BioSpin) operating at X-band $(9.4 \mathrm{GHz})$ equipped with an ELEXSYS Super High Sensitivity Probehead, 200G rapid scan coils and helium gas flow system (ESR900, Oxford Instruments). Each individual sample with TOAC was prepared as follows: A $3 \mathrm{~mm}$ OD/1 mm ID quartz tube (Aachener Quarz-Glas Technologie Heinrich, Germany) was filled with $8 \mu \mathrm{L}$ of the cell extract and put in the spectrometer. $2 \mu \mathrm{L}$ of TOAC were added and mixed using a Hamilton syringe. Reduction kinetics was studied for the concentration range $0.4-20 \mathrm{mM}$ of nitroxide. Kinetic curves were monitored over $2 \mathrm{~min}$ in a $2 \mathrm{D}$-experiment (abscissa 1 - field; abscissa 2 - time) with rapid scan coils (1 spectrum/s). Parameters for rapid-scan experiment were: Sweep ramp-up $=500 \mathrm{~ms}$, Sweep ramp-down $=10 \mathrm{~ms}$, Sweep delay $=10 \mathrm{~ms}$, Time constant $=1.28 \mathrm{~ms}$. The amount of nitroxide species in the probe was determined by double integration of the spectrum.

All measurements were performed at $18^{\circ} \mathrm{C}$. Each kinetic curve was measured three times.

\section{Acknowledgments}

This project was financially supported by the DFG (DR 743/2-1), Zukunftskolleg University of Konstanz (DFG-ZUK 52/1), and the Konstanz Research School Chemical Biology. We thank J. S. Hartig for stimulating discussions.

\section{References}

[1] E. Zavoisky, Spin-magnetic resonance in paramagnetics, J. Phys. E 9 (1945) 245-249.

[2] B. Commoner, J. Townsend, G.E. Pake, Free radicals in biological materials, Nature 174 (1954) 689-691.

[3] B. Commoner, J.J. Heise, J. Townsend, Light-induced paramagnetism in chloroplasts, Proc. Natl. Acad. Sci. U.S. 42 (1956) 710-718.

[4] H. Hu, G. Sosnovsky, H.M. Swartz, Simultaneous measurements of the intraand extra-cellular oxygen concentration in viable cells, Biochim. Biophys. Acta 1112 (1992) 161-166.

[5] H.J. Halpern, Stable soluble paramagnetic compounds, in: L.J. Berliner (Ed.), In vivo EPR (ESR) Theory and applications, Kluwer Academic/Plenum Publishers, New York, 2003. pp. 207-209.

[6] B.L. Bales, R.A. Blum, D. Mareno, M. Peric, H.J. Halpern. Solvent and temperature dependence of the hyperfine coupling constants in CTPO. J. Magn. Reson. 98 (1992) 299-307.

[7] J.F.W. Keana. M.J. Acarregui, S.L.M. Boyle, 2,2-Disubstituted-4.4dimethylimidazo-linidinyl-3-oxy nitroxides: Indicators of aqueous acidity through variation of $\mathrm{a}_{\mathrm{N}}$ with $\mathrm{pH}$. J. Am. Chem. Soc. 104 (1982) 827-830.

[8] E.J. Husted, J.]. Kirchner, A. Spaltenstein, P.B. Hopkins, B.H. Robinson, Monitoring DNA dynamics using spin-labels with different independent monitoring DNA dynamics using spin-labels

[9] K. Chen, P.D. Morse II, H.M. Swartz, Kinetics of enzyme-mediated reduction of lipid soluble nitroxide spin labels by living cells, Biochim. Biophys. Acta 943 (1988) $477-484$.

[10] O. Schiemann, N. Piton, J. Plackmeyer, B.E. Bode, T.F. Prisner, J.W. Engels, Spin labeling of oligonucleotides with the nitroxide TPA and use of PELDOR, a pulse EPR method, to measure intramolecular distances, Nat. Protoc. 2 (2007) 904EPR n.

[11] S. Domingo Köhler, A. Weber, S.P. Howard, W. Welte, M. Drescher, The prolinerich domain of TonB possesses an extended polyproline II-like conformation of sufficient length to span the periplasm of Gram-negative bacteria, Protein Sci. 19 (2010) 625-630.

[12] M. Robotta, P. Braun, B. van Rooijen, V. Subramaniam, M. Huber, M. Drescher Direct evidence of coexisting horseshoe and extended helix conformations of membrane-bound alpha-synuclein, ChemPhysChem 12 (2011) 267-269.

[13] R.J. Usselman, E.D. Walter, D. Willits, T. Douglas, M. Young, D.J. Singel, Monitoring structural transitions in icosahedral virus protein cages by sitedirected spin labeling, J. Am. Chem. Soc. 133 (2011) 4156-4159.

[14] G. Bacic, M.J. Nilges, R.L. Magin, T. Walczak, H.M. Swartz, In vivo localized ESR spectroscopy reflecting metabolism. Magn. Reson. Med. 10 (1989) 266-272.

[15] S. Colacicchi, M. Ferrari, A. Sotgiu, In vivo electron paramagnetic resonance spectroscopy/imaging: first experiences, problems, and perspectives, Int. J Biochem. 24 (1992) 205-214.

[16] R. Igarashi, T. Sakai, H. Hara, T. Tenno, T. Tanaka, H. Nochio, M. Shirakawa, Distance determination in proteins inside Xenopus laevis oocytes by double electron-electron resonance experiments, J. Am. Chem. Soc. 132 (2010) $8228-$ 8229.

[17] I. Krstic, R. Hänsel, O. Romainczyk, J.W. Engels, V. Dötsch, T.F. Prisner, Longrange distance measurements on nucleic acids in cells by pulsed EPR spectroscopy, Angew. Chem. Int. Ed. 50 (2011) 5070-5074

[18] M. Azarkh, O. Okle, V. Singh, I.T. Seemann, J.S. Hartig, D.R. Dietrich, M. Drescher, Long-range distance determination in a DNA model system inside Xenopus laevis oocytes by in-cell spin-label EPR, ChemBioChem (2011). doi: $10.1002 /$ cbic. 201100281 .

[19] P. Selenko, Z. Serber, B. Gadea, J. Ruderman, G. Wagner, Proc. Natl. Acad. Sci USA 103 (2006) 11904-11909.

[20] R. Hänsel, S. Foldynová-Trantirková, F. Löhr, J. Buck, E. Bongartz, E. Bamberg, H. Schwalbe, V. Dötsch, L. Trantirek, Evaluation of parameters critical for observing nucleic acids inside living Xenopus laevis oocytes by in-cell NMR spectroscopy, J. Am. Chem. Soc. 131 (2009) 15761-15768.

[21] L.J. Berliner, G.R. Eaton, S.S. Eaton (Eds.), Distance Measurements in Biological Systems by EPR, Biological Magnetic Resonance, Vol. 19, KLUWER, New York 2000.

[22] M.M. Dedmon, C.N. Patel, G.B. Young, C.G. Pielak, FlgM gains structure in living cells, Proc. Natl. Acad. Sci. USA 99 (2002) 12681-12684.

[23] I.B. Dawid, T.D. Sargent, Xenopus laevis in developmental and molecular biology, Science 240 (1988) 1443-1448.

[24] J.D. Richter, A Comparative Methods Approach to the Study of Oocytes and Embryos, Oxford University Press, New York, 1999.

[25] E.A. Barnard, R. Miledi, K. Sumikawa, Translation of exogenous messenger RNA coding for nicotinic acetylcholine receptors produces functional receptors in Xenopus oocytes, Proc. R. Soc. Lond. B: Biol. Sci. 215 (1982) 241-246.

[26] M. Mishina, T. Kurosaki, T. Tobimatsu, Y. Morimoto, M. Noda, T. Yamainoto, M Terao, J. Lindstrom, T. Takahashi, M. Kuno, S. Numa, Expression of functional acetylcholine receptor from cloned cDNAs, Nature 307 (1984) 604-608.

[27] S.M. Jarvis, D.A. Griffith, Expression of the rabbit intestinal N2 Na+/nucleoside transporter in Xenopus laevis oocytes, Biochem. J. 278 (1991) 605-607. 
[28] A.G. Swick, M. Janicot, T. Cheneval-Kastelic, J.C. McLenthian, M.D. Lane, Promoter-cDNA-directed heterologous protein expression in Xenopus laevis oocytes, Proc. Natl. Acad. Sci. USA 89 (5) (1992) 1812-1816.

[29] A.M. Shafer, T. Kálai, S.Q. Bin Liu, K. Hideg, J.C. Voss, Site-specific insertion of spin-labeled L-amino acids in Xenopus oocytes, Biochemistry 43 (2004) 84708482.

[30] N. Kocherginsky, H.M. Swartz, Nitroxide Spin Labels: Reactions in Biology and Chemistry, CRC, Boca Raton, 1995.

[31] V. Singh, M. Azarkh, T.E. Exner, J.S. Hartig, M. Drescher, Human telomeric quadruplex conformations studied by pulsed EPR, Angew. Chem. Int. Ed. 48 (2009) 9728-9730.

[32] L.D. Belmont, A.A. Hyman, K.E. Sawin, T.J. Mitchison, Real-time visualization of cell cycle-dependent changes in microtubule dynamics in cytoplasmic extracts, Cell 62 (1990) 579-589.

[33] J.J. Blow, R.A. Laskey, Initiation of DNA replication in nuclei and purified DNA by a cell-free extract of Xenopus eggs, Cell 47 (1986) 577-587.
[34] M.J. Lohka, J.L. Maller, Induction of nuclear envelope breakdown, chromosome condensation, and spindle formation in cell-free extracts, J. Cell. Biol. 101 (2) (1985) $518-523$

[35] L. Michaelis, M.L. Menten, Die Kinetik der Invertinwirkung, Biochem. Z. 49 (1913) 333-369.

[36] H.M. Swartz, M. Sentjurc, P.D. Morse II, Cellular metabolism of water-soluble nitroxides: effect on rate of reduction of cell/nitroxide ration, oxygen concentrations and permeability of nitroxides, Biochim. Biophys. Acta 888 concentrations and permeability of nitroxides, Biochim. Biophys. Acta 888 (1986) 82-90

[37] M.A. Savageau, Enzyme kinetics in vitro and in vivo: Michaelis-Menten revisited, Prin. Med. Biol. 4 (1) (1995) 93-146.

[38] W.H. de Ronde, B.C. Daniels, A. Mugler, N.A. Sinitsyn, I. Nemenman, Mesoscopic statistical properties of multistep enzyme-mediated reactions, IET Syst. Biol. 3 (2009) 429-437. 